\title{
Absceso del iliopsoas: Claves para el diagnóstico imagenológico
}

\author{
Leonardo Lidid A.', Juan Salvador Casas M. ${ }^{2}$
}

1. Profesor Asistente de Radiología. Universidad de Chile, Facultad de Medicina. Servicio de Radiología. Hospital San Juan de Dios. Radiólogo. Servicio de Radiología. Hospital DIPRECA. Santiago, Chile.

2. Radiólogo. Servicio de Radiología. Hospital San Juan de Dios. Universidad de Chile, Facultad de Medicina. Santiago, Chile.

\section{Iliopsoas abscess: Imaging keys to accurate diagnosis}

\begin{abstract}
Although rare, iliopsoas abscess has a high rate of morbidly and mortality. Its diagnosis is difficult given the numerous etiologies and varied clinical presentation. Objective: To discuss the causes, dissemination pathways, differential diagnoses and management of iliopsoas abscess; emphasizing the important role of CT and MR through multiple imaging examples. Conclusion: Imagenological analysis is essential to determine complexity, extention, and anatomical origin of this condition, fundamental parameters in the prognosis and management of these patients.
\end{abstract}

Keywords: Iliopsoas, Abscess, Retroperitoneum, Computer tomography, Magnetic resonance imaging, Management.

Resumen. Si bien es infrecuente, el absceso del músculo iliopsoas exhibe una alta tasa de morbilidad y mortalidad. Su diagnóstico es difícil debido a las numerosas etiologías y su variada presentación clínica. Objetivo: Discutir las causas, vías de diseminación, diagnósticos diferenciales y manejo del absceso del iliopsoas, poniendo especial énfasis en el rol de la tomografía computada y la resonancia magnética en su diagnóstico. Conclusión: La evaluación con estudios de imagen es esencial para determinar la complejidad, extensión y origen anatómico de esta patología, parámetros que son fundamentales para determinar el pronóstico y el manejo en este tipo de pacientes.

Palabas clave: Iliopsoas, Absceso, Retroperitoneal, Tomografía computada, Resonancia magnética

Lidid L, et al. Absceso del iliopsoas: Claves para el diagnóstico imagenológico. Revi Chil Radiol 2017; 23(4): 176-186. Correspondencia: Leonardo Lidid Alonso / leolidid@gmail.com

Trabajo enviado el 25 de octubre de 2017. Aceptado para publicar el 09 de diciembre de 2017.

\section{Introducción}

El absceso del iliopsoas, aunque infrecuente, es una entidad potencialmente letal, insidiosa y de difícil diagnóstico, estando su caracterización dada principalmente por reportes y series de casos $^{1,2}$. El cuadro clínico es inespecífico, por lo que la sospecha inicial es a menudo escasa. La triada clásica de presentación (fiebre, dolor lumbar y cojera) se observa tan solo en un $30 \%$ de los casos, dificultándose aún más en la actualidad su diagnóstico, por el enmascaramiento de la sintomatología generado por el indiscriminado uso de antibióticos $3,4,5,6$.

Ante tal escenario, los estudios de imagen se han convertido en un pilar diagnóstico fundamental, tanto por la precocidad de su detección como por la especificidad alcanzada por algunas técnicas.

El objetivo de este artículo es describir las principales características imagenológicas del absceso del iliopsoas tanto en tomografía computada (TC) como en resonancia magnética (RM), haciendo especial énfasis en sus causas, rutas de diseminación y diagnósticos diferenciales.

Anatomía: Compartimento del iliopsoas y vías de diseminación retroperitoneal.

Si bien los abscesos pueden presentar fistulización a estructuras y órganos adyacentes (Figura 1), tanto las colecciones como las alteraciones inflamatorias 
suelen respetar las barreras anatómicas normales, observándose habitualmente una extensión longitudinal de éstos a través de los planos fasciales. Por estas razones, el conocimiento de la anatomía del compartimento del iliopsoas y de los espacios retroperitoneales, es esencial para la comprensión de las vías de diseminación y compromiso a distancia de esta patología $a^{7,8}$.

El compartimento del músculo iliopsoas corresponde al espacio anatómico extraperitoneal delimitado bajo las fascias del músculo psoas mayor, psoas menor y músculo ilíaco. Los músculos psoas mayor y psoas menor se originan de los procesos transversales de la $12^{a}$ vértebra torácica y vértebras lumbares. Desde este punto, las fibras descienden por debajo del ligamento arcuato del diafragma para entrar en el retroperitoneo abdominal y pelviano. En la pelvis, el
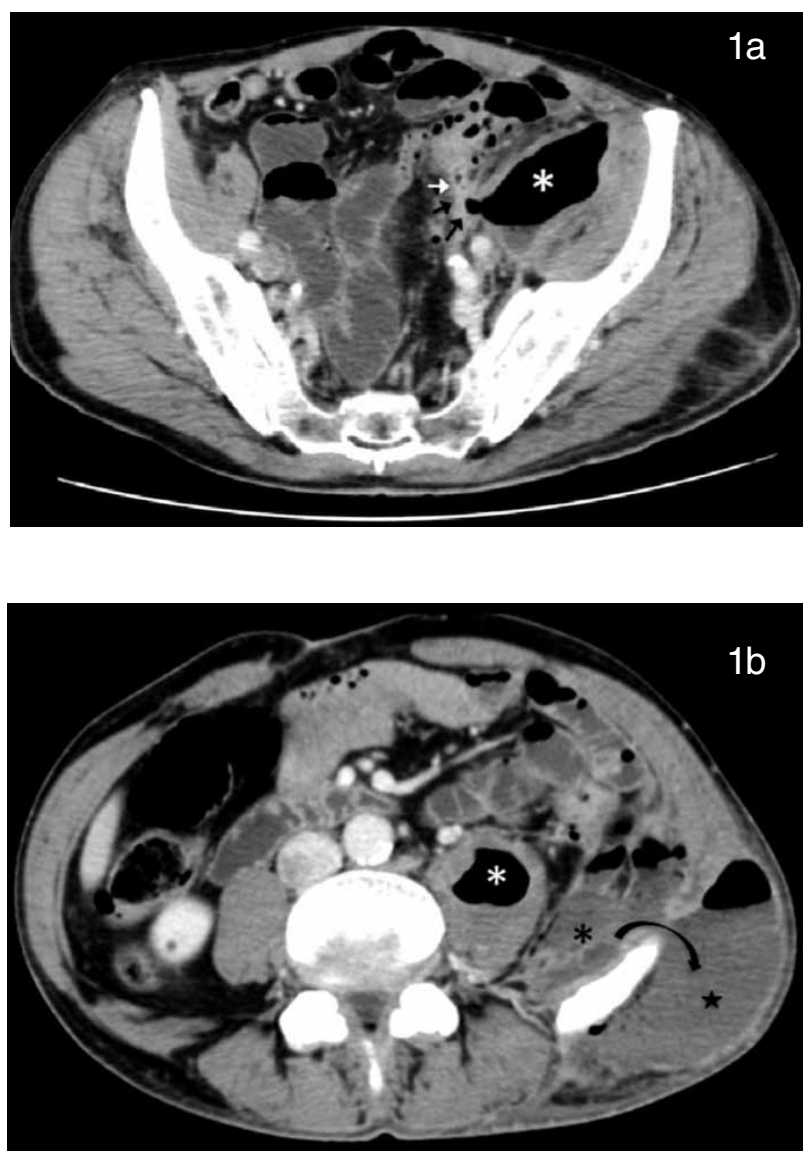

Figura 1. Hombre de 64 años con diverticulitis complicada y absceso de iliopsoas con formación de fístula en región glútea y lumbar. a) TC con contraste de zona pélvica, que demuestra cambios inflamatorios pericolónicos y fistulización de un divertículo (flechas) hacia la cavidad de un absceso del iliopsoas izquierdo con gas en su interior (asterisco). b) Reconstrucción multiplanar axial oblicua de TC que muestra la extensión del absceso del iliopsoas (asteriscos) a través de una fístula (flecha curva) hacia el plano lumbar y glúteo ipsilateral (estrella). músculo ilíaco surge del aspecto profundo de la cresta ilíaca para unirse al músculo psoas bajo el ligamento inguinal, alcanzando después de este punto el muslo proximal, donde se observa su inserción a nivel del trocánter menor del fémur (Figura 2). De esta forma se establece un potencial canal de comunicación que abarca desde el tórax (Figura 3) hasta la cadera y el muslo (Figuras 4 y 5$)^{7,9,10}$.

Además de lo anterior, este compartimento tiene

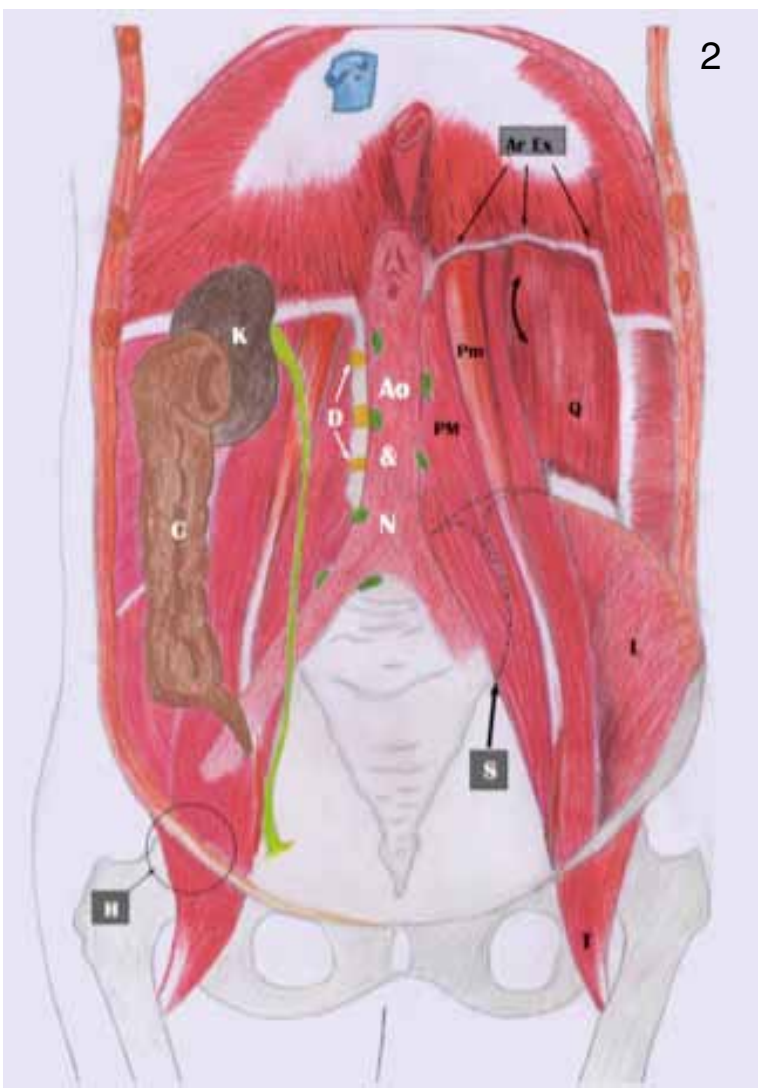

Figura 2. Músculo lliopsoas: esquema de relaciones. $A$ la izquierda del dibujo se representa la pared abdominal posterior con una escisión de los ligamentos arcuatos del diafragma ( $\operatorname{Ar} E x)$. A este nivel se observa una extensión torácica retrofrénica (flecha curvada) de los músculos cuadrado lumbar (Q), psoas mayor (PM) y psoas menor $(P m)$. El músculo ilíaco $(L)$ en el hueso ilíaco desciende para formar el tendón conjunto (T) con el músculo psoas mayor hacia su inserción femoral. El psoas menor (un músculo comúnmente ausente) se inserta por separado en la eminencia iliopectínea del hueso ilíaco. A la derecha se representaron las principales relaciones del músculo iliopsoas (marcadas con letras blancas): riñón y uréter (K), colon y apéndice $(C)$, discos vertebrales (D), aorta y linfonodos cercanos $($ Ao \& N), cadera $(H)$ y articulación sacroilíaca (S) (ambos detrás del iliopsoas distal). La articulación sacroilíaca se representa a izquierda con una línea de puntos. Cuando se infectan, todas estas localizaciones son potenciales causas de formación de abscesos del iliopsoas secundarios. 

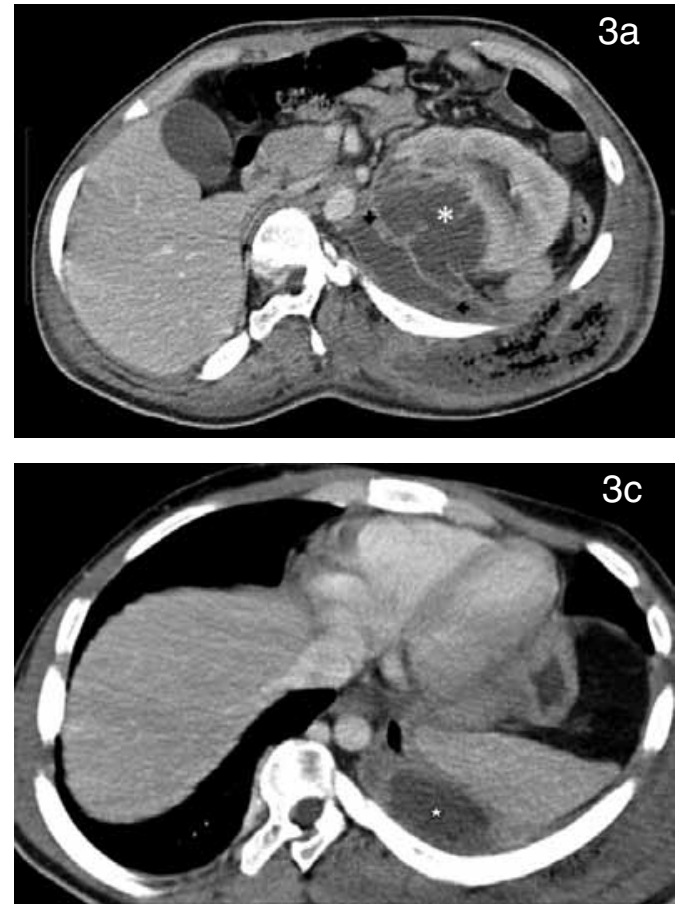

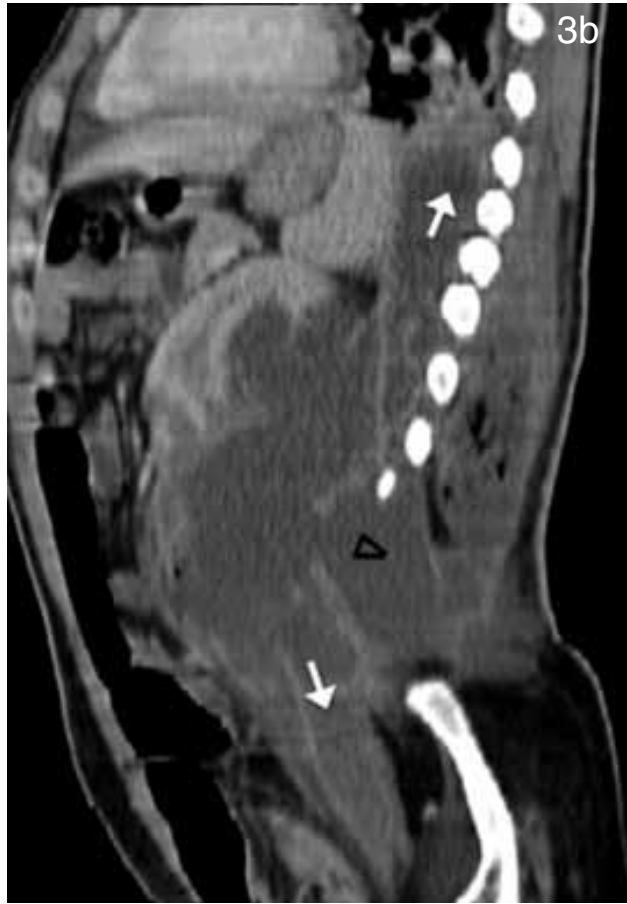

Figura 3. Hombre de 22 años en postración, con escoliosis congénita y un absceso del iliopsoas de origen renal con compromiso de la transición toracoabdominal por vía retrofénica. a) Imagen axial de TC contrastado de abdomen que muestra un absceso renal (asterisco) con extensión al compartimento del iliopsoas ipsilateral (flechas negras). b) Reconstrucción sagital de TC contrastado, que muestra diseminación caudal y cefálica de la colección (flechas blancas) a través de este mismo compartimento. También se aprecia fistulización subcutánea hacia la región lumbar (punta de flecha). c) Imagen axial de TC contrastado del segmento toracoabdominal que muestra la extensión del absceso del compartimento del iliopsoas (estrella) a cefálico por vía retrofrénica, tal como se muestra en b. Cf. Figura 2.
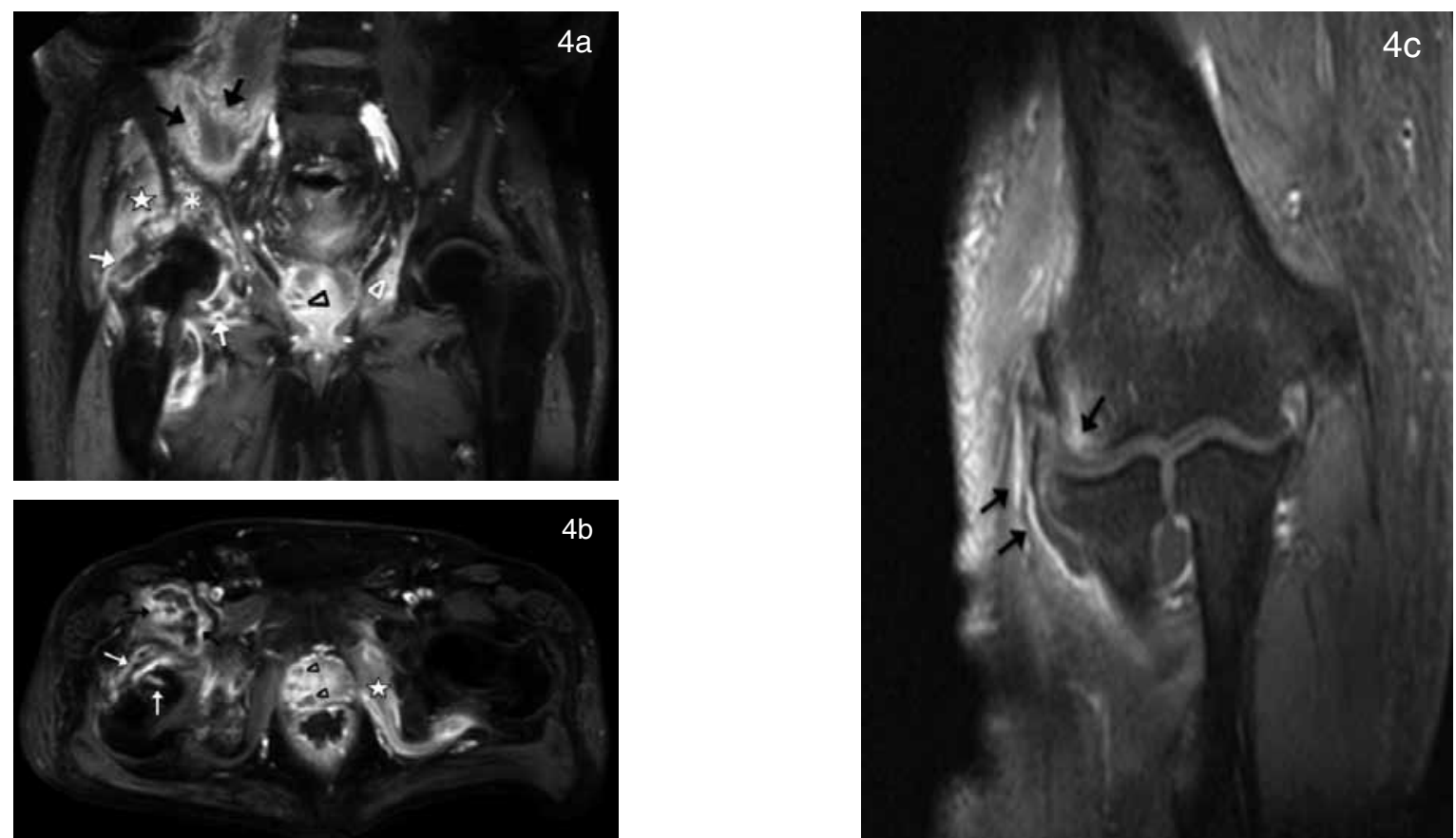

Figura 4. Hombre de 63 años con absceso primario del iliopsoas y metástasis séptica causada por bacteriemia complicada por Staphylococcus aureus. Se descartó endocarditis. Por dolor e impotencia funcional de piema derecha se realiza estudio de imágenes. a) Imagen coronal contrastada ponderada en T1 a nivel de la pelvis, que revela impregnación periférica en una colección del iliopsoas derecho, con características de absceso (flechas negras). Debido a la vecindad anatómica hay artritis séptica de la cadera (flechas blancas), osteomielitis ilíaca (asterisco) y miositis glútea ipsilateral (estrella). Como resultado de la diseminación hematógena se observa un absceso prostático (cabeza de flecha negra) y piomiositis del obturador intemo contralateral (cabeza de flecha blanca). b) Imagen axial con contraste ponderada en T1 de la pelvis que muestra de forma más clara los abscesos prostáticos (cabezas de flecha) y la piomiositis del obturador interno izquierdo (estrella blanca). Extensión distal del absceso del iliopsoas (flechas negras) con afectación por contigüidad de la cadera derecha (artritis séptica) (flechas blancas). Finalmente, esta complicación determinó la pérdida total de la articulación. c) Imagen coronal con contraste ponderada en $T 1$ del codo izquierdo, con artritis séptica hematógena (flechas). Adicionalmente, la mano también presentaba celulitis. 

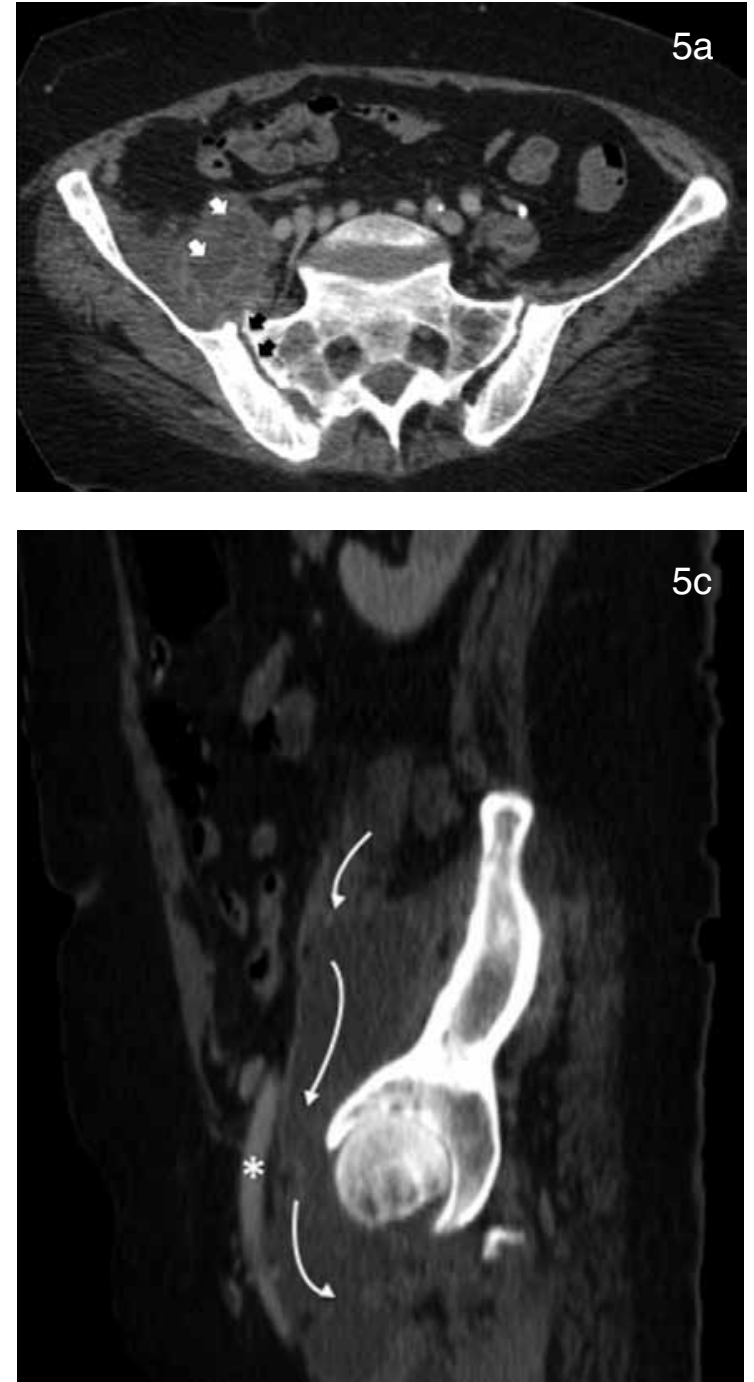

una estrecha relación anatómica con el retroperitoneo, especialmente con los espacios perirrenal y pararrenal posterior, ya que la fascia renal posterior se fusiona con la fascia del músculo iliopsoas (y del cuadrado lumbar) en múltiples puntos, determinando así potenciales vías de comunicación entre ambas estructuras. Considerando que las fascias renales anterior y posterior no siempre están fusionadas en su parte superior o inferior, estas aberturas potenciales también ofrecen una ruta de diseminación tanto hacia el mediastino (dada su conexión con los hiatos diafragmáticos a través del área desnuda del hígado); como hacia los espacios extraperitoneales pélvianos, respectivamente ${ }^{7,11}$.

Hacia ventral, el músculo iliopsoas está estrechamente relacionado con el páncreas, la aorta, la vena cava inferior y los ganglios linfáticos retroperitoneales. Estructuras como el duodeno, ciego y apéndice en el lado derecho del abdomen, y el colon descendente en el lado izquierdo, están cerca del compartimento del iliopsoas (Figura 2). Por tanto, la erosión de los planos fasciales retroperitoneales por procesos infecciosos que los afecten puede dar lugar a lesiones

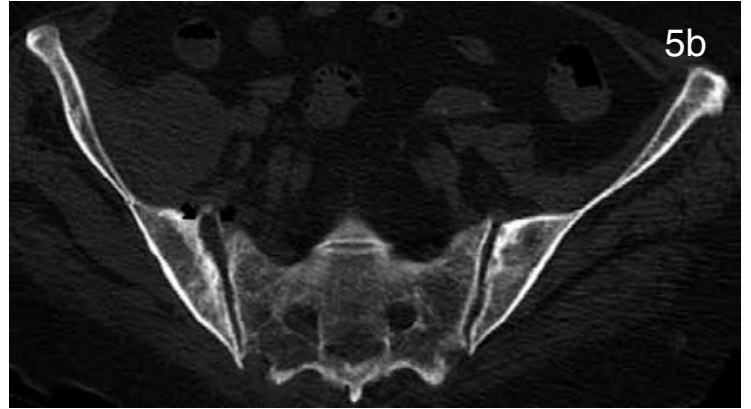

Figura 5. Hombre de 81 años con absceso del iliopsoas originado por sacroileitis séptica. a) Corte axial de TC contrastado a nivel pélvico en ventana de partes blandas que muestra un absceso del iliopsoas derecho (flecha blanca), que se origina de la articulación sacroilíaca adyacente (flecha negra). b) Corte axial de TC contrastado en ventana ósea que muestra erosiones y ensanchamiento del espacio articular consistente con sacroileitis séptica derecha (flechas). c) Reconstrucción sagital oblicua de TC contrastado en ventana de partes blandas que muestra la extensión caudal de un absceso hacia el muslo y cadera a través de la región inguinal (flechas). Vasos femorales (asterisco).

dentro de este compartimento, como se describirá más adelante?.

\section{Etiología}

De acuerdo a su etiología, los abscesos del iliopsoas se pueden dividir en primarios (sin claro foco anatómico de origen) o secundarios (con origen anatómico identificable).

La frecuencia relativa varía según la zona geográfica, siendo los abscesos primarios predominantes en India, Hong Kong y Sudáfrica (sobre un 95\% de los casos), en tanto que el tipo secundario es predominante en Europa y representa aproximadamente un $80 \%$ de los casos 5 . Sin embargo, la frecuencia de los abscesos primarios pareciera estar en aumento en occidente, especialmente en pacientes inmunodeprimidos, y en particular en población con $\mathrm{VIH}^{4}$.

En los abscesos primarios el modelo explicativo está dado por la presencia de bacteriemia desde focos ocultos hacia un músculo iliopsoas con un daño celular previo, que suele corresponde a una lesión de tipo traumático no reportada por el paciente. A pesar de que el mecanismo no está del todo claro, patologías como la piomiositis tropical ${ }^{5,12}$ o entidades como las bacteriemias complicadas (de descripción más reciente) (Figura 4) pudieran explicar la generación de un compromiso muscular séptico hematógeno desde una fuente a distancia ${ }^{13,14}$. El principal agente etiológico en occidente es el Staphylococcus aureus $s^{4,6,15}$, aunque infecciones por las especies Pseudomonas, Haemophilus y Proteus también han 
sido documentadas ${ }^{16}$. Los pacientes típicos incluyen niños, adultos inmunocomprometidos (p. ej., diabetes mellitus, inmunosupresión, SIDA, insuficiencia renal) o usuarios de drogas intravenosas ${ }^{4,6}$.

En los abscesos de tipo secundario se produce una afectación por vecindad del iliopsoas por extensión de la patología desde un órgano adyacente. Las principales etiologías de los abscesos son de origen gastrointestinal, musculoesquelético y genitourinario, aunque otros orígenes como fuentes vasculares también han sido descritas (Figura 6). Las patologías específicas principalmente involucradas se describen en la (Tabla 1). Si bien hay variaciones en la literatura respecto al orden de frecuencia de las distintas especies, los microorganismos más frecuentemente implicados siguen siendo las bacterias entéricas y el Staphyloccous aureus s, $^{2,5,6,15}$.

La tuberculosis como causa secundaria de abs-
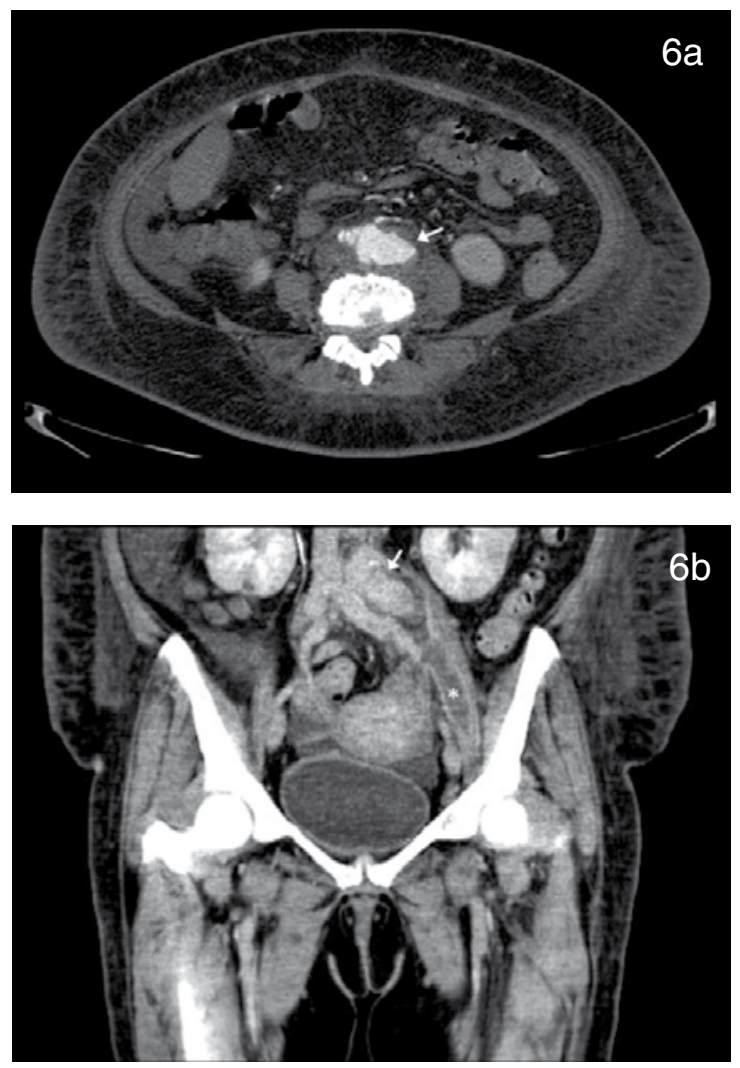

Figura 6. Mujer de 52 años con absceso de iliopsoas causado por aneurisma micótico secundario a embolia séptica en el contexto de una endocarditis infecciosa por Streptococcus viridans. a) Corte axial de TC contrastado en fase arterial que muestra un aneurisma micótico de la arteria ilíaca común izquierda (flecha) con cambios inflamatorios alrededor de ella. b) Corte axial de TC contrastado en fase portovenosa que muestras un aneurisma micótico (flecha) y formación de absceso secundario del iliopsoas (asterisco) con realce inflamatorio de la pared. [Reproducido a partir de la referencia 23. International free publishing license. Símbolos añadidos]. ceso del iliopsoas ha ido decreciendo por la mejora global en las condiciones de salud; sin embargo, se ha observado un reciente resurgimiento de esta patología por el incremento de la población con $\mathrm{VIH}$. Además de lo anterior, este diagnóstico diferencial también debería ser considerado en aquellos lugares donde esta enfermedad aún no ha sido completamente erradicada, especialmente en algunos países en desarrollo, en los cuales la tuberculosis vertebral (Mal de Pott) todavía es considerada como la causa más común de absceso del iliopsoas. En alrededor de un $5 \%$ de casos de Mal de Pott se puede desarrollar este tipo de abscesos ${ }^{8,17}$.

\section{Hallazgos de imagen}

La pérdida del contorno del iliopsoas o la presencia de gas en el espacio retroperitoneal constituyen hallazgos radiográficos clásicos de absceso del iliopsoas, pero carecen de sensibilidad. En algunos casos también es posible detectar la causa especialmente en aquellos casos de origen esquelético; sin embargo, éstos constituyen hallazgos tardíos ${ }^{16,18}$.

La ecografía puede ser útil en la evaluación de colecciones intramusculares; sin embargo, presenta una limitada utilidad en la evaluación de estructuras retroperitoneales profundas debido a la anatomía del paciente (p.ej., obesidad) y al hecho de que es un examen muy dependiente de la técnica utilizada por el operador. El Ultrasonido es diagnóstico en aproximadamente un $60 \%$ de los casos de absceso del iliopsoas ${ }^{8,16}$.

La TC y la RM ofrecen la exactitud diagnóstica

Tabla 1. Etiologías de abscesos del iliopsoas.

\begin{tabular}{|l|l|}
\hline Tipo de abceso & Origen \\
\hline Primarios & $\begin{array}{l}\text { Bacteriemia desde un foco } \\
\text { oculto. }\end{array}$ \\
\hline Secundarios & $\begin{array}{l}\text { Gastrointestinal: Enf. de } \\
\text { Chron, diverticulitis, apendicitis } \\
\text { aguda complicada entre otros. } \\
\text { Genitourinario: Pielonefritis } \\
\text { complicadas. } \\
\text { Musculoesquelético: Discitis } \\
\text { infecciosa, sacroilieitis infec- } \\
\text { ciosa, artritis séptica de cadera } \\
\text { entre otros. } \\
\text { latrogénicos: Complicacio- } \\
\text { nes quirúrgicas (abdominales/ } \\
\text { esqueléticas) o por instrumen- } \\
\text { talización (vascular/urológica). } \\
\text { Otros: Linfoadenitis supurativa, } \\
\text { aortitis infecciosa, procesos } \\
\text { inflamatorios pélvicos entre } \\
\text { otros. }\end{array}$ \\
\hline
\end{tabular}


más alta, con una sensibilidad comparable entre 80 y $100 \%{ }^{19,20}$. Entre ambos métodos, la TC aparece como especialmente útil por su mayor disponibilidad y menor costo ${ }^{16}$. Aun así, algunos investigadores han propuesto la superioridad de la RM por su habilidad de discriminar tejidos blandos y de visualizar los abscesos sin administración de medio de contraste, ofreciendo de esta forma, una solución para pacientes con contraindicación en el uso de dichos agentes ${ }^{6,4}$ (Figura 7).

En ambos métodos, los hallazgos traducen los mismos fenómenos de base, los cuales son dependientes del estadio de progresión de la enfermedad.

En las fases iniciales los hallazgos son relativamente inespecíficos ${ }^{20}$ y corresponden a la presencia de miositis del iliopsoas del lado afectado, que se caracteriza por aumento de tamaño, edema e hiperemia del músculo comprometido. El edema y la hiperemia son principalmente identificables por RM como una señal aumentada en secuencias sensibles al líquido e impregnación muscular del medio de contraste, respectivamente. Por otro lado, el aumento de tamaño muscular puede ser fácilmente evidenciable en ambos métodos de estudio.

En la fase de abscedación ambas técnicas son útiles, revelando signos de piomiositis con zonas de licuefacción muscular central y realce periférico del medio de contraste. Las áreas de licuefacción son
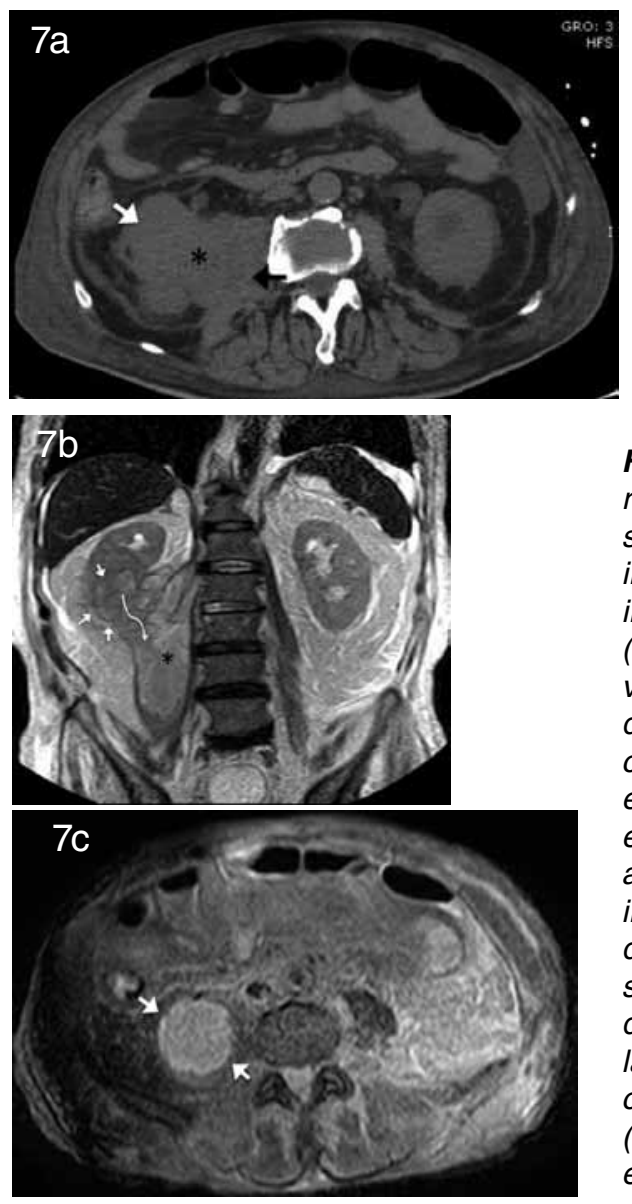

Figura 7. Hombre de 63 años con absceso del iliopsoas secundario a absceso renal, que ingresa con síndrome infeccioso. Ventaja diagnóstica de la RM sobre TC en estudios sin uso de contraste (en este caso contraindicado por insuficiencia renal). a) Corte axial de TC abdominal no contrastado que revela imagen masiforme (asterisco) del riñón derecho (flecha blanca) e iliopsoas (flecha negra). Aunque se sospecha un absceso, una lesión tumoral sólida podría verse de similar aspecto. b) Imagen coronal, potenciada en T2 del abdomen que revela una lesión renal moderadamente hiperintensa (flechas rectas) en continuidad (flecha sinuosa) con un compartimento del iliopsoas derecho expandido (asterisco) que tiene la misma señal. Hay una relativa hiperseñal en el resto del abdomen debido a edema retroperitoneal y ascitis. c) Imagen axial, con supresión de grasa ponderada en T2 del abdomen que muestra un iliopsoas derecho más grande (flechas blancas). Aunque hay inhomogeneidad del campo magnético, la hiperseñal presente en este compartimiento es sugerente de líquido. d) Imagen potenciada en difusión (b1000), mismo nivel que en A. Como se esperaba, hay menos artefactos y mejor delineación de la lesión (flechas) con esta secuencia. La alta intensidad de señal exhibida se correlaciona con la baja señal en el mapa de coeficiente de difusión aparente (no mostrado) que confirma una restricción en la difusión. En este contexto, este hallazgo es consistente con contenido purulento. 


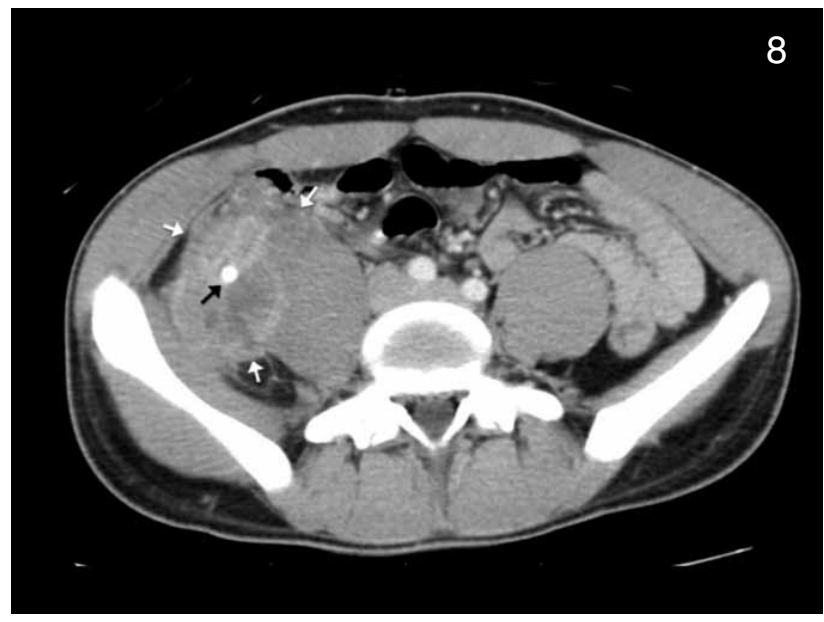

Figura 8. Hombre de 19 años con absceso de iliopsoas secundario a apendicitis aguda. TC contrastado de la pelvis muestra un absceso del iliopsoas (flechas blancas) causado por inflamación apendicular secundaria a la impactación de un apendicolito (flecha negra).
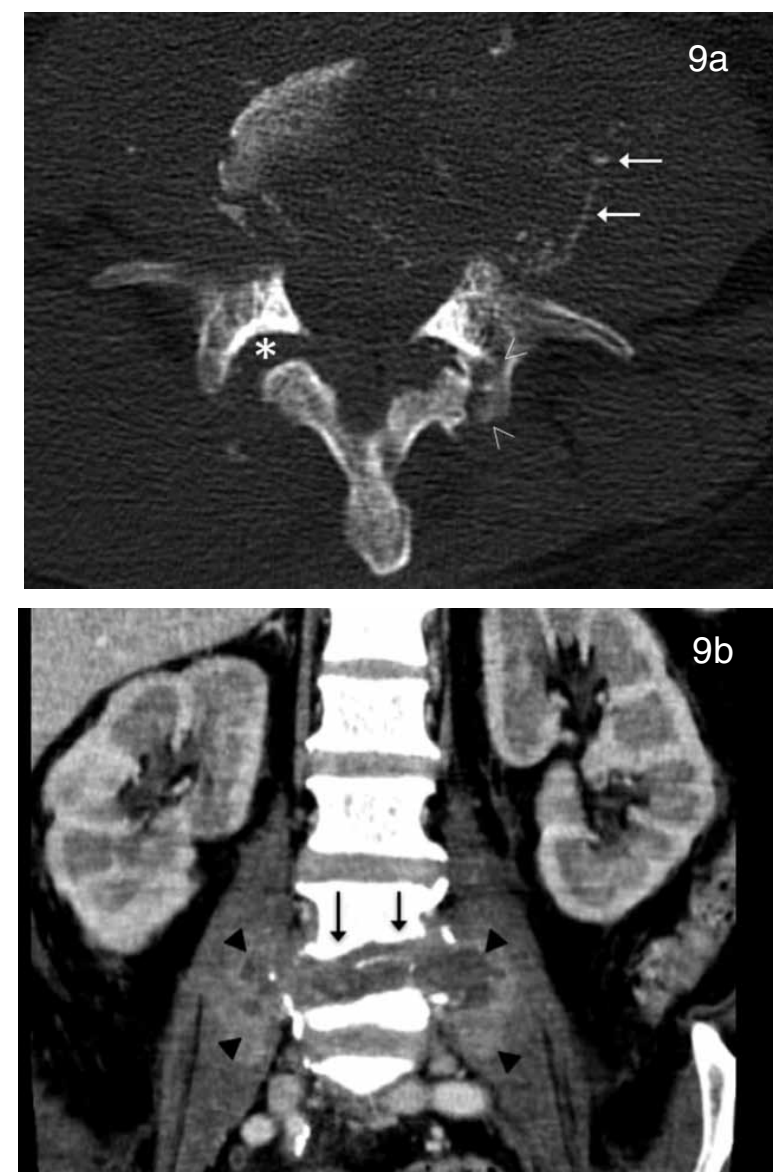

Figura 9. Hombre de 55 años con espondilodiscitis bacteriana. a) Corte axial de TC no contrastado de columna lumbar en ventana ósea que revela destrucción del cuerpo vertebral con múltiples fragmentos óseos (flechas). También se observa compromiso posterior (articulación interapofisiaria) con ensanchamiento articular (asterisco) y erosiones óseas (cabezas de flecha). b) Reconstrucción coronal de TC contrastado de abdomen en ventana de partes blandos que muestra abscesos de ambos iliopsoas (cabezas de flecha) debidos a la discitis ya descrita (flechas). logía tuberculosa de estas alteraciones se describen algunos signos relativamente específicos para su diagnóstico. En el caso de la espondilodiscitis estos signos corresponden a una relativa preservación de la amplitud del espacio discal, con una afectación multisegmentaria (generalmente del segmento torácico), y un compromiso vertebral predominantemente anterior (por diseminación contigua subligamentaria entre cuerpos vecinos. Junto a estos también se describe la presencia de abscesos paraespinales con prominentes calcificaciones y un marcado anillo periférico de impregnación (Figura 10). En el caso de la linfoadenitis tuberculosa estos signos corresponden a la tendencia a formar conglomerados adenopáticos y sobre todo a la existencia de una marcada hipodensidad ganglionar asociada a una impregnación periférica en forma de anillo (Figura 11). En ausencia de signos específicos locales de esta patología, la afectación categórica de otros parénquimas (p.ej., patrón pulmonar miliar) pudieran orientar a el diagnóstico $24,25,26$.

Si bien los estudios de medicina nuclear (p.ej., exploración con citrato de Galio-67) tienen cierto grado de utilidad en el diagnóstico de patología inflamatoria/infecciosa, en general su uso ha sido limitado a la detección de ese tipo de abscesos en la evaluación de pacientes con fiebre de origen desconocido o pacientes con siembra séptica (habituales de observar en embolias sépticas o bacteriemias complejas), donde el uso de 18F-FDG PET/TC ha desempeñado un papel cada vez más prominente en los últimos años ${ }^{13,19,27,28,29}$.

\section{Diagnósticos Diferenciales}

Existen dos consideraciones diagnósticas diferenciales principales para el absceso del iliopsoas: los tumores retroperitoneales y los hematomas.

En las neoplasias retroperitoneales los principales problemas diagnósticos surgen con el linfoma y los tumores con extensa necrosis (Figura 12). Si bien la ausencia de un contexto infeccioso permite plantear más fácilmente estos diagnósticos, otros elementos que sugieren un origen neoplásico son la presencia de adenopatías, nódulos sólidos captantes (especialmente en el aspecto periférico de la lesión), erosión tumoral ósea e infiltración (o ausencia de respeto) de los limites compartimentales fasciales normales ${ }^{7,8}$.

En el caso del hematoma no infectado del iliopsoas, el diagnóstico puede ser difícil ya que puede ser indiferenciable de cualquier otra colección líquida (en particular los hematomas crónicos). Sin embargo, los siguientes parámetros pueden orientar a este diagnóstico: ausencia de cambios inflamatorios de la grasa adyacente, tendencia (en estadios precoces) a una afectación difusa de todo el compartimento muscular, detección del sitio de 

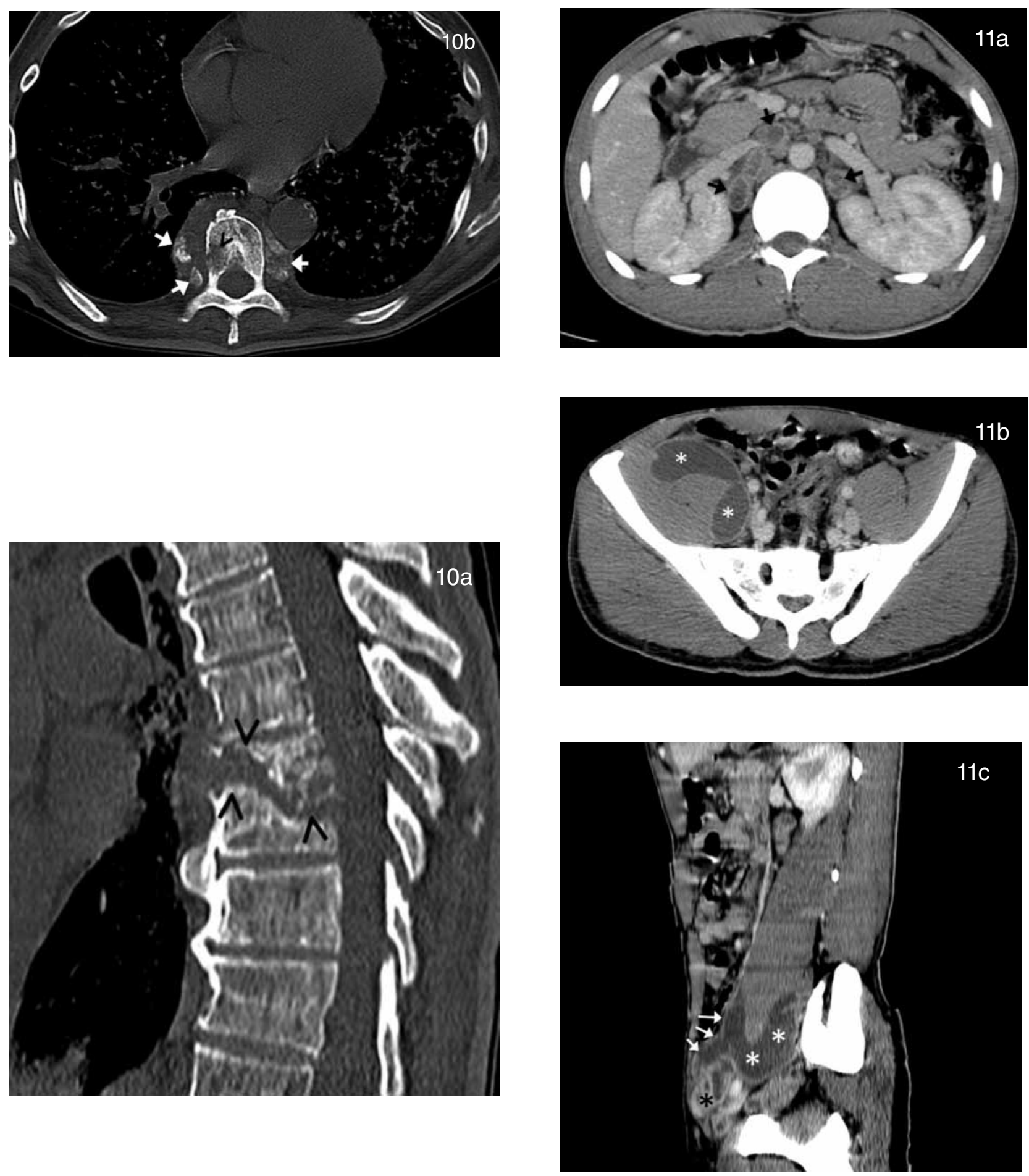

Figura 10. Hombre de 74 años con espondilodiscitis tuberculosa. a, Reconstrucción sagital TC de tórax en ventana ósea (detalle a nivel de la columna). Destrucción ósea que afecta los cuerpos vertebrales (cabezas de flecha). No hay compromiso de los elementos posteriores. Cf. Figura 6. b, Corte axial de TC no contrastado de tórax en ventana ósea que muestra signos característicos de espondilodiscitis tuberculosa con engrosamiento de tejidos blandos paravertebrales con calcificaciones gruesas (flechas) y áreas de destrucción ósea (cabeza de flecha). Si bien no es la ventana ideal, se puede observar el compromiso pulmonar de la tuberculosis, más extenso a izquierda.

Figura 11. Hombre de 30 años de edad, con VIH/SIDA con linfadenitis y absceso tuberculoso del iliopsoas derecho. a) Corte axial de TC contrastado de abdomen que muestra múltiples linfoadenopatías hipodensas con realce periférico en anillo (flechas negras); aspecto característico del compromiso tuberculoso. b) Corte axial de TC contrastado de pelvis que muestra absceso del iliopsoas derecho (asteriscos). c) Reconstrucción sagital oblicua contrastada de TC de abdomen-pelvis a la altura de la ingle derecha que muestra un conglomerado adenopático (asterisco negro) que drena espontáneamente su contenido (flechas) en el compartimento del iliopsoas (asteriscos blancos). 

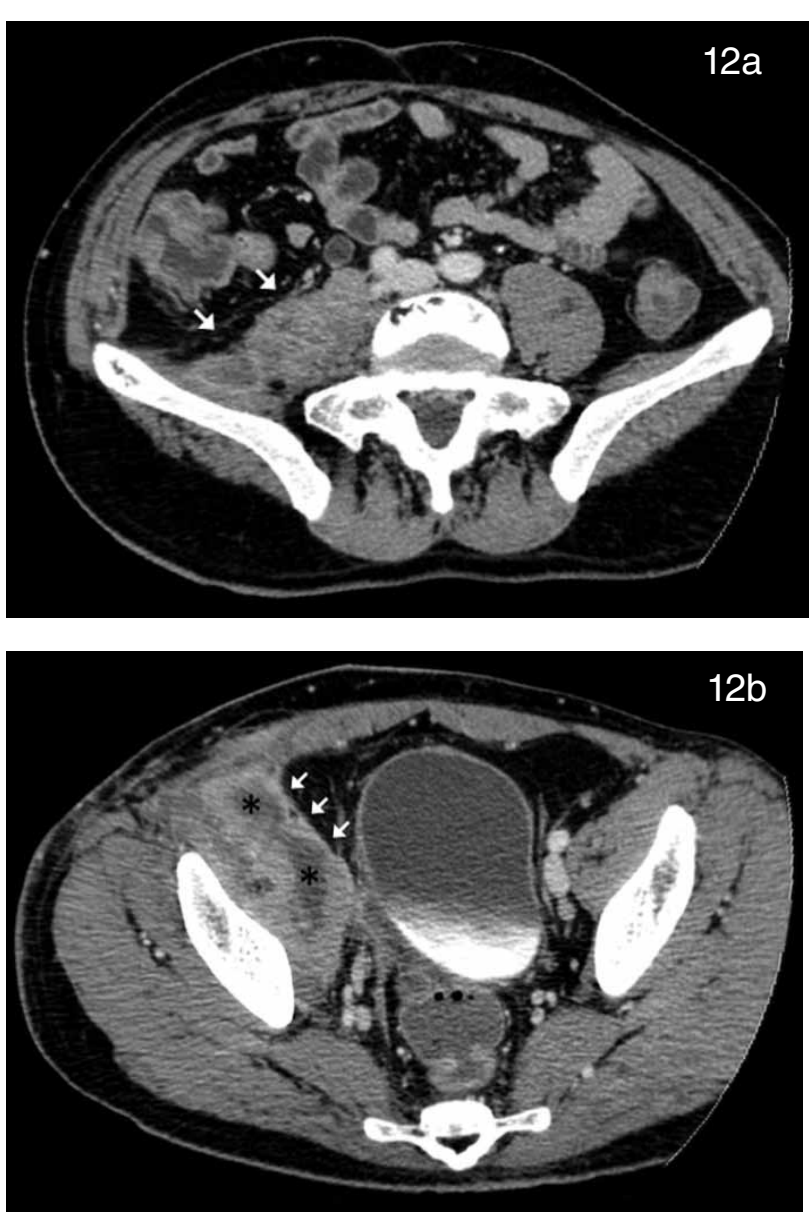

Figura 12. Hombre de 35 años $\mathrm{VIH}(+)$ en tratamiento antirretroviral, con masa inguino-abdominal derecha de rápido crecimiento, que experimenta evolución febril. Biopsia de ganglio linfático inguinal: linfoma no Hodgkin de células $B$ grandes. a, Corte axial de TC contrastado de pelvis que muestra una masa retroperitoneal a derecha con impregnación periférica del medio de contraste que parece comprometer el compartimento del músculo iliopsoas ipsilateral (flechas blancas). $b$, Corte axial de TC en un plano más caudal que el anterior, mostrando un área hipodensa central sugerente de contenido líquido (asteriscos) en la masa del iliopsoas derecho (flechas blancas). Al momento de la biopsia se drenó líquido que fue negativo para microorganismos.

sangramiento activo en las adquisiciones contrastadas, presencia de niveles eritrocitarios o de hiperdensidad/ intensidad (en el estudio tomográfico sin contraste y secuencias potenciadas en $\mathrm{T} 1$, respectivamente), sumado a los antecedentes de caída brusca en los niveles de hematocrito en un contexto traumático o en un paciente con una alteración conocida de la coagulación (Figura 13) 7,8 .

Adicionalmente existe un signo, especialmente detectable en estudios de TC, que puede aportar otra clave para el diagnóstico: la presencia de gas dentro del músculo (en ausencia de instrumentalización previa) tiene una fuerte asociación con procesos sépticos alcanzando casi el $50 \%$ de estos casos, especialmente en infecciones de etiología anaeróbica (Figura 1) ${ }^{8,10,30}$.

Si aún todo esto fuera insuficiente, se puede realizar una aspiración/biopsia guiada por imágenes con análisis microbiológico y patológico para poder llegar finalmente al diagnóstico correcto ${ }^{7,8}$.
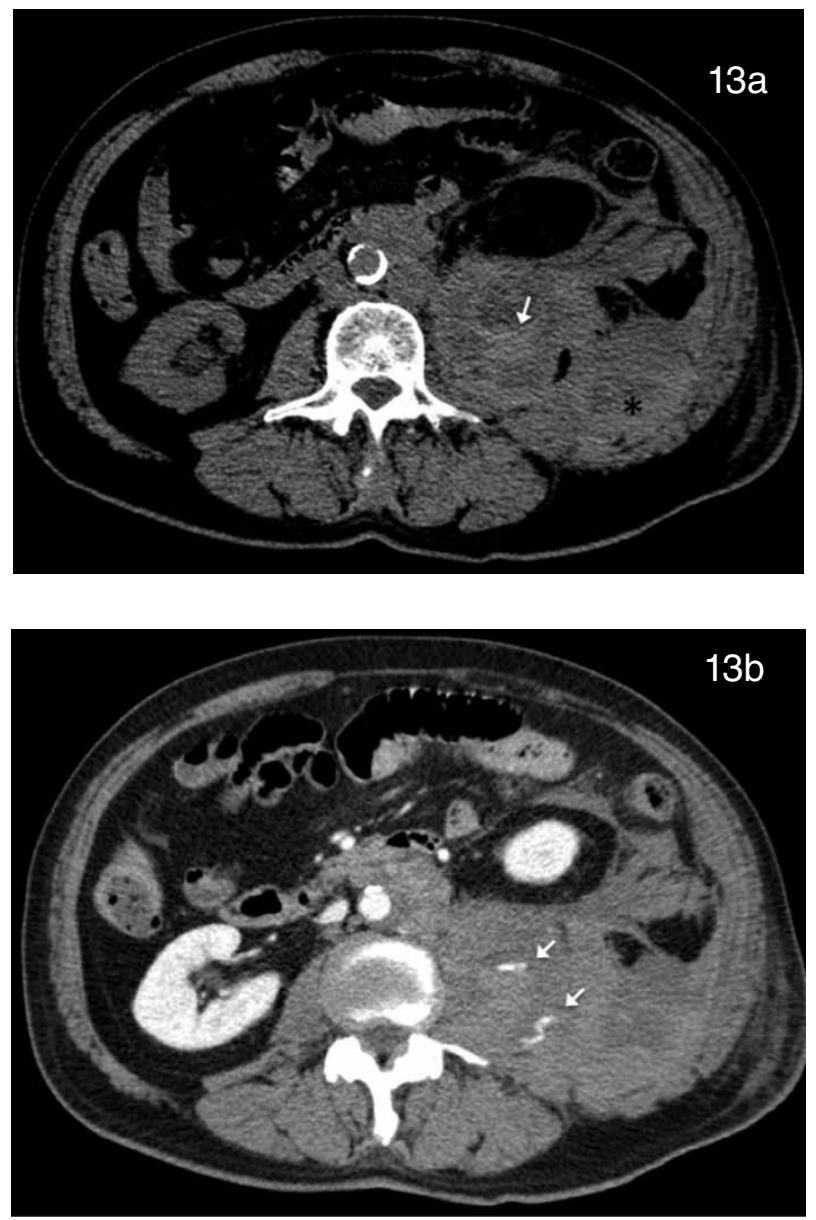

Figura 13. Hombre de 79 años con cáncer de próstata, en terapia anticoagulante por trombosis venosa, con caída del hematocrito. Hematoma del iliopsoas izquierdo. a) Corte axial de TC abdominal sin contraste que muestra un aumento de volumen del iliopsoas izquierdo, con nivel hematocrito (flecha) y un componente espontáneamente denso en su interior (asterisco). b) Corte axial de TC con contraste (mismo nivel), con sitios de sangrado activo (flechas); uno de ellos corresponde al nivel descrito en a.

\section{Manejo}

El manejo de esta patología se basa en dos pilares fundamentales: cobertura antibiótica (según los perfiles microbiológicos) y drenaje por vía quirúrgica o percutánea (en abscesos sobre los $3 \circ 3,5 \mathrm{~cm}$ ). Actualmente, la modalidad de elección es la última, debido a los buenos resultados y las menores tasas de complicación ${ }^{30,31}$. La opción quirúrgica (utilizada sola o en combinación con técnicas percutáneas) queda reservada para los abscesos multiloculados, con ex- 
tenso compromiso de estructuras y para los abscesos secundarios a algunas patologías específicas entre las que destacan la enfermedad inflamatoria intestinal, la pielonefritis (xantogranulomatosa o enfisematosa), los aneurismas micóticos, las neoplasias perforadas y en los abscesos epidurales con compresión espinal, entre otros $30,32,33,34,35$.

\section{Pronóstico}

La tasa de mortalidad de esta enfermedad está en directa relación con la morbilidad del paciente, el tipo de absceso (primario o secundario) y la patología que lo causa. En la literatura se ha reportado una tasa de mortalidad de un $18,9 \%$ causada por abscesos secundarios del iliopsoas, frente a una tasa de mortalidad del $5 \%$ debida a complicaciones relacionadas con abscesos primarios, siendo los abscesos de origen intestinal los que presentan el peor pronóstico. Estos son valores cambiantes en la literatura, alcanzando en algunas series una mortalidad hospitalaria de hasta el $44 \%$ en presencia de organismos formadores de gas y hasta el $100 \%$ en pacientes sépticos con abscesos no drenados oportunamente ${ }^{5,30}$.

\section{Conclusiones}

El absceso del iliopsoas es una enfermedad rara e insidiosa con signos y síntomas clínicos inespecíficos, en los que la TC y la RM juegan un rol crucial para el diagnóstico oportuno. La evaluación imagenológica es esencial para determinar el lugar de origen, la extensión anatómica y la complejidad de los abscesos involucrados. Estas características repercuten tanto en el pronóstico como en el manejo del paciente, ya sea de tipo médico, intervencional o quirúrgico.

\section{Conflicto de Intereses}

No hay conflictos de intereses.

Fondos: No se requirireron fondos para este estudio.

\section{Agradecimientos}

Queremos dar la gracias a Fernando Carreño, Mario Zerega, Sebastián Yévenes, Felipe Zumaeta, y al resto de los radiológos del Hopital San Juan de Dios por compartir sus conocimientos, y hallazgos con sus colegas; así como también agradecer a los Becados, ya que, con sus preguntas, su trabajo e interés nos hacen descubrir y aprender algo nuevo cada día.

\section{Referencias}

1. Capitán Manjón C, Tejido Sánchez A, Piedra Lara JD, et al. Retroperitoneal Abscesses. Scand J Urol Nephrol 2003; 37: 139-144.

2. Fernández-Ruiz M, Estébanez-Muñoz M, López-Medrano F, Aguado J.M. Absceso del músculo iliopsoas: Tratamiento y evolución en una serie de 35 pacientes. Enferm Infecc Microbiol Clin 2012; 30(6): 307-311.

3. Liu Y, Huang W, Huang T, Kunin CM. Inappropriate use of antibiotics and the risk for delayed admission and masked diagnosis of infectious diseases: a lesson from Taiwan. Arch Intern Med 2001; 161(19): 2366-2370.

4. Shields D, Robinson P, Crowley TP. lliopsoas abscess: A review and update on the literature. Int J Surg 2012; 10(9): 466-469.

5. Ricci M, Rose F, Meyer K. Pyogenic psoas abscess: Worldwide variations in etiology. World J Surg 1986; 10(5): 834-843.

6. Mallick I, Thoufeeq M. H, Rajendran T. lliopsoas abscesses. Postgrad Med J 2004; 80 (946): 459-462.

7. Muttarak M, Peh WC. CT of unusual iliopsoas compartment lesions. Radiographics 2000; 20 (Spec No): 53-S66.

8. Leão AR de S, Amaral RPG, Abud TG, Demarchi GTS, Filho F, Oliveira E de, et al. Patologias do compartimento iliopsoas: Avaliação radiológica. Radiol bras 2007; 267-272.

9. Heng Tan Ch, Vikram R, Boonsirikamchai P, Faria S, Charnsangavej $\mathrm{Ch}$, Bhosale P. Pathways of extrapelvic spread of pelvic disease: imaging findings. RadioGraphics 2011; 31: 117-133.

10. Warshauer D, Lee J, Patel H. Retroperitoneum. In: Lee J, ed. Computed Body Tomography with MRI Correlation. 4th ed. Philadelphia: Lippincott Williams \& Wilkins; 2006:1155-1232.

11. Lidid L, Valenzuela J, Villaroel C, Alegria J. Crossing the barrier: when the diaphragm is not a limit. AJR 2013; 200:62-70.

12. Chattopadhyay B, Mukhopadhyay M, Chatterjee A, Biswas PK, Chatterjee N, Debnath NB. Tropical pyomyositis. North Am J Med Sci 2013; 5:600-603.

13. Mitchell D, Howden P. Diagnosis and management of Staphylococcus aureus bacteraemia. Intern Med J 2005; 35(2):S17-S24.

14. Lin M, Rezai K, Schwartz D. Septic pulmonary emboli and bacteremia associated with deep tissue infections caused by community-acquired methicillin-resistant Staphylococcus aureus. J Clin Microbiol. 2008; 46(4):1553-1555.

15. Kim Y, Hoon Yoon J, Kim S, Wie S, Kim Y. Etiology and outcome of iliopsoas muscle abscess in Korea; changes over a decade. Int J Surg 2013; 11(10): 1056-1059.

16. Garner J, Meiring P, Ravi K, Gupta R. Psoas abscess: not as rare as we think? Colorectal Dis 2007; 9(3): 269-274.

17. Chawla K, D'Souza A, Bhat S, Mukhopadhayay Ch. Primary tubercular psoas abscess: a rare presentation. J Infect Dev Ctries 2012; 6(1): 86-88.

18. Gouliouris T, Aliyu S, Brown N. Spondylodiscitis: update on diagnosis and management. J Antimicrob Chemother 2010; 65 Suppl 3: iii11-iii24.

19. Yang RH, Chu YK. Gallium- 67 citrate scan in the early assessment of psoas muscle abscess. Radiology Case Reports. (Online) 2015; 10(2); 839.

20. Takada T, Terada K, Kajiwara H, Ohira Y. Limitations of using imaging diagnosis for psoas abscess in its early stage. Intern Med 2015; 54(20): 2589-2593.

21. Moore WA, Khatri G, Madhuranthakam AJ, Sims RD, Pedrosa I. Added value of diffusion-weighted acquisitions in MRI of the abdomen and pelvis. AJR 2014; 202(5): 995-1006.

22. Subhawong TK, Jacobs MA, Fayad LM. Diffusionweighted MR imaging for characterizing musculoskeletal lesions. RadioGraphics 2014; 34: 1163-1177. 
23. Cocio R, Zérega M,Yévenes S, Lidid L, et al (2016, Jan 13). Septic embolism and embolic source, what and how to look for?. Eurorad. http://www.eurorad.org/case. php?id=13289. http://dx.doi.org/10.1594/EURORAD/ CASE.13289

24. Burrill J, Williams C, Bain G, Conder G, Hine A, Misra R. Tuberculosis: A radiologic review. RadioGraphics 2007; 27: 1255-1273.

25. Lee K. Comparison of pyogenic spondylitis and tuberculous spondylitis. Asian Spine J 2014; 8(2): 216-223.

26. Mohapatra $P$, Janmeja $A$. Tuberculous lymphadenitis. J Assoc Physicians India 2009; 57 (6): 585-590.

27. Vos F, et al. Metastatic infectious disease and clinical outcome in Staphylococcus aureus and Streptococcus species bacteremia. Medicine 2012; 91(2): 86-94.

28. Kao PF, Tzen KY, Tsui KH, Tsai MF, Yen TC. The specific gallium-67 scan uptake pattern in psoas abscesses. Eur J Nucl Med 1998; 25(10): 1442-1447.

29. Haroon A, Zumla A, Bomanji J. Role of fluorine 18 fluorodeoxyglucose positron emission tomographycomputed tomography in focal and generalized infectious and inflammatory disorders. Clin Infect Dis 2012; 54(9): 1333-1341.

30. Hsieh MS, et al. Features and treatment modality of iliopsoas abscess and its outcome: A 6-year hospitalbased study. BMC Infect Dis 2013; 13: 578.

31. Yacoub WN, et al. Psoas abscess rarely requires surgical intervention. Am J Surg 2008; 196(2): 223-227.

32. Hsu R, Lin F. Psoas abscess in patients with an infected aortic aneurysm. J Vasc Surg 2007; 46: 230-235.

33. Yang J, Lee J, Cha S, Joo Y. Psoas abscess caused by spontaneous rupture of colon cancer. Clin Orthop Surg 2011; 3: 342-344.

34. Kudalkar D, Remé P, Cunha B, Brook M, Brook S. Xanthogranulomatous pyelonephritis complicated by psoas abscess. Heart Lung® 2004; 33:339-42.

35. Craig W, Wagner B, Travis M. Pyelonephritis: Radiologicpathologic review. RadioGraphics 2008; 28: 255-276. 